\title{
Reply to the letter by Filik and Biyikoglu regarding the hypothesis that changes in the extracellular matrix incurred by systemic hernia disease (herniosis) are hostile to the development of colon cancer
}

\author{
R. C. Read
}

Received: 27 September 2010/ Accepted: 15 October 2010/Published online: 1 December 2010

(c) Springer-Verlag 2010

These gastrointestinal investigators from Turkey [1] are the first to provide evidence that this hypothesis [2] may actually be fact. Their data, obtained from 435 consecutive patients who in 2009 underwent total colonoscopy, showed that, whereas 35 had diverticulosis and 24 had cancer, only two had both. In the latter, neoplasms arose in the rectum. These patients were smokers, suffering an addiction known to induce malignancy. Further support for this hypothesis can be gleaned from research conducted by Ghajar and Bissell [3] on cancer of the breast, the most common malignancy in women. They point out that the ECM in the mammary gland, which comprises $80 \%$ of its tissue, was once thought only to provide infrastructure but is now known to be a key component of the cellular micro- environment that influences genetic expression. They maintain that in other organs (kidney, skin, lung, colon, and ovary), the genome is similarly influenced by the ECM.

\section{References}

1. Filik L, Biyikoglu I (2010) [letter] Colon cancer and diverticula: a single-institution experience. Hernia 14:453

2. Read RC (2010) Systemic hernia disease protects against cancer: an hypothesis. Hernia 14(2):119-121

3. Ghajar CM, Bissell MJ (2008) Extracellular matrix control of mammary gland morphogenesis and tumorigenesis: insights from imaging. Histochem Cell Biol 130:1105-1118
R. C. Read ( $₫)$

University of Arkansas for Medical Sciences,

304 Potomac Street, Rockville, MD 20850, USA

e-mail: read@post.harvard.edu 